\title{
Clinical Outcome of IM-Guided Total Knee Arthroplasty with Inappropriate Femoral Resection in Coronal Plane
}

Young-Wan Moon, MD, Jeong-Hoon Han, MD, Keun-Ho Lee, MD, Sung Won Jang, MD, and Jai-Gon Seo, MD Department of Orthopedic Surgery, Samsung Medical Center, Sungkyunkwan University School of Medicine, Seoul, Korea

Purpose: The purpose of this study was to evaluate clinical results and accuracy of femoral cutting in the coronal plane in total knee arthroplasty (TKA) using a fixed length intramedullary guide.

Materials and Methods: From 2005 to 2008, 101 patients (154 knees) underwent TKA (NexGen LPS implant). The minimal follow-up period was 3 years (mean, 4.4 years). The patients were divided into two groups (group 1, $94 \leq \alpha$ angle $<98$; group 2, 94>a, $98 \leq \alpha$ ). Mechanical axis deviation (MAD), patellar tilting angle (PTA), Knee Society Knee Score (KSKS), and Knee Society Function Score (KSFS) were measured in both groups.

Results: There were 120 knees in group 1 and 34 knees in group 2. There was no significant intergroup difference in the postoperative MAD (group 1, $1.59^{\circ}$; group $\left.2,1.91^{\circ}\right)$. The number of outliers with $\geq 2^{\circ}$ MAD was 65 in group 1 and 24 in group 2 . The mean PTA, KSKS, and KSFS were $10.17^{\circ}, 96.0$, and 96.6, respectively, in group 1 and $11.58^{\circ}, 84.5$, and 85.5 , respectively, in group 2 .

Conclusions: The percentage of coronal alignment outliers was relatively high (34 in 154 cases, 22\%) after TKA using a fixed length intramedullary guide. However, there was no statistically significant intergroup difference in clinical results (KSKS, $\mathrm{p}=0.67$; KSFS, $\mathrm{p}=0.56$ ).

Keywords: Total knee arthroplasty, Intramedullary guide, Mechanical axis deviation, Alpha angle

\section{Introduction}

Traditionally, femoral and tibial cuts in total knee arthroplasty (TKA) have been made perpendicular to the mechanical axis of the knee using either an intramedullary (IM) or an extramedullary alignment guide system. However, there is no consensus on the differences and relative superiority of the two systems ${ }^{1,2)}$. Hungerford ${ }^{3)}$ and Hungerford and Krackow ${ }^{4)}$

Received June 13, 2012; Revised (1st) July 6, 2012;

(2nd) September 4, 2012; Accepted October 8, 2012

Correspondence to: Young-Wan Moon, MD

Department of Orthopedic Surgery, Samsung Medical Center,

Sungkyunkwan University School of Medicine, 81 Irwon-ro, Gangnamgu, Seoul 135-710, Korea

Tel: +82-2-3410-3509, Fax: +82-2-3410-0061

E-mail: ywmoon@skku.edu

This is an Open Access article distributed under the terms of the Creative Commons Attribution Non-Commercial License (http://creativecommons.org/licenses/by-nc/3.0/) which permits unrestricted non-commercial use, distribution, and reproduction in any medium, provided the original work is properly cited. suggested that the proximal tibia be cut in $3^{\circ}$ of varus since tibial plateau is in $3^{\circ}$ varus relative to the tibial axis and the distal femur in $3^{\circ}$ of valgus with respect to the mechanical axis. Their method is advantageous for restoring the biomechanics of the knee. However, it has been of limited use because of the likelihood of damage to the lateral collateral ligament or the popliteus tendon caused by excessive varus cutting of the tibia or extensive resection of the lateral femoral condyle. On the other hand, in TKA using an IM alignment guide system, the use of a fixed length IM rod that cannot be adjusted to the length of the femur during surgery may cause a gap between the medullary canal and the rod, and thus affect the femoral component position. In particular, deep insertion of the rod for additional femoral bone resection in knees with flexion contracture often results in abnormal placement of the femoral component witha angle under 94 or over 98 . In this study, we investigated the relationship between inappropriate femoral resection in the coronal plane and clinical outcome in IM-guided TKA. 


\section{Materials and Methods}

\section{Materials}

Of the patients who underwent TKA using the NexGen legacy posterior stabilised (LPS; Zimmer, Warsaw, IN, USA) and an IM alignment guide system for $6^{\circ}$ of valgus correction, 101 patients (154 knees) who were available for $\geq 3$ years of follow-up between April 2005 and May 2008 (mean, 4.4 years; range, 3 to 6.8 years) were included in this study. The exclusion criteria were $\geq 6^{\circ}$ of valgus correction and $<3$ years of follow-up period. There were 5 males and 96 females with a mean age of 69.64 years (range, 53 to 85 years).

\section{Surgical Technique}

The knee joint was exposed through the medial capsular incision. The anterior and posterior cruciate ligaments were removed and soft tissue release was performed. After soft tissue balancing, distal femoral resection was performed using an intramedullary cutting guide, which was followed by the proximal

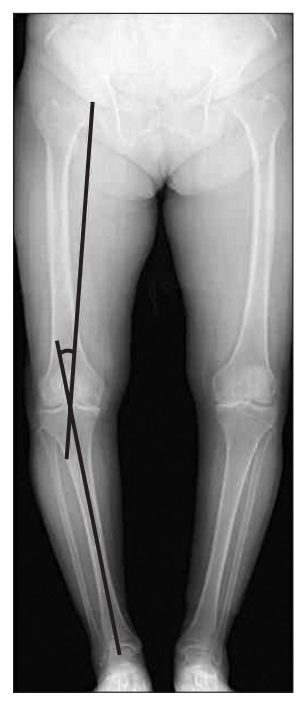

Fig. 1. Mechanical axis deviation. tibial cut using an extramedullary guide. An intramedullary rod for distal femoral cut was inserted in $6^{\circ}$ of valgus so that the cutting guide was positioned perpendicular to the mechanical axis of the femur. After resection of the anterior aspect of the distal femur, distal femoral cut and proximal tibial cut were performed using a device that allows for creation of a rectangular extension gap until medial-lateral and flexion-extension gaps were balanced. Other procedures were identical to those used in conventional TKA. All the operations were performed by the same surgeon. Patellar resurfacing was performed in only 10 patients (13 knees).

\section{Clinical and Radiographic Assessments}

On the clinical assessment, American Knee Society Knee Score (KSKS) and American Knee Society Functional Score (KSFS) were evaluated preoperatively and at the last follow-up.

On the radiographic assessment, mechanical axis deviation (MAD) that was defined as the angular difference between the

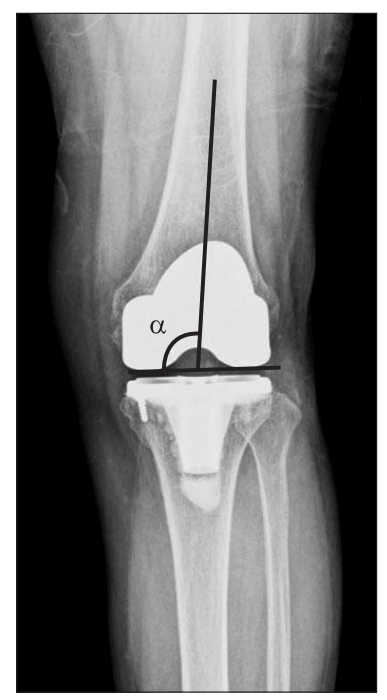

Fig. 3. The angle between the mechanical axis of the femur and the anatomical axis ( $\alpha$ angle).
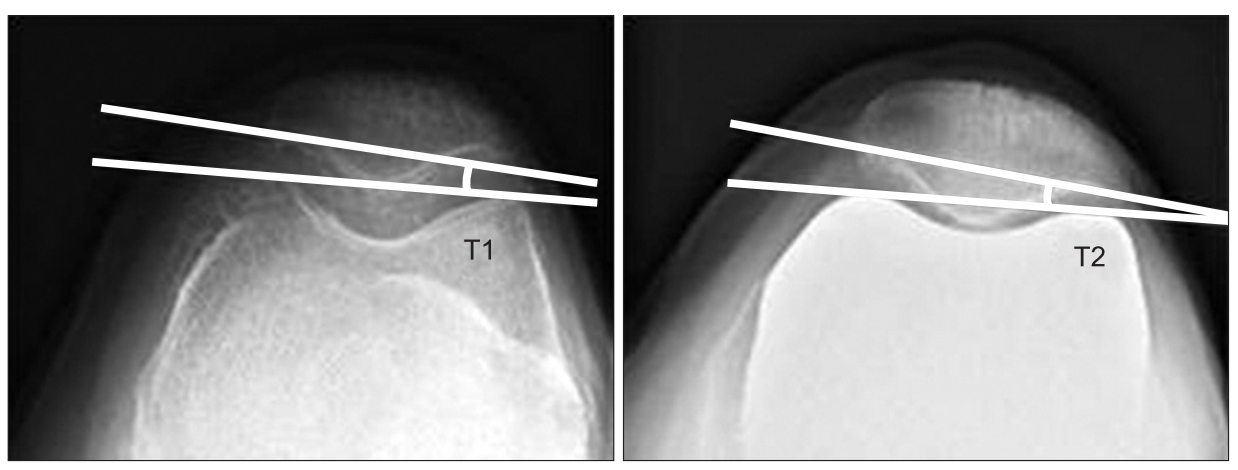

Fig. 2. Patellar tilt angle. 
femoral and tibial mechanical axes was measured on the long leg anteroposterior (AP) radiograph (Fig. 1) preoperatively and at the last follow-up. Patellar tilt angle (PTA) on the merchant view was measured preoperatively and at the last follow-up (Fig. 2).

In addition, the angle created by the mechanical axis and anatomical axis of the femur was measured on the preoperative long leg AP radiograph to assess the adequacy of $6^{\circ}$ of valgus insertion of the intramedullary rod. All the radiographic measurements were performed by the same orthopedic surgeon.

\section{Statistical Analysis}

The location of the femoral component ( $\alpha$ angle) was assessed on the postoperative plain radiograph (Fig. 3). Based on previous studies, ${ }^{5,6)}$, we arbitrarily took $2^{\circ}$ as an acceptable margin of error for $\alpha$ angle. The patients were divided according to the $\alpha$ angle into two groups: group $1,94 \leq \alpha$ angle $<98$ and group 2, $94>\alpha$, $98 \leq \alpha$. Intergroup differences in the MAD, PTA, KSKS, KSFS, and pre- and postoperative changes for each parameter were analyzed. The relationship between the $\alpha$ angle and the MAD, PTA, KSKS, and KSFS, and the cut-off value that yields the greatest clinical and radiological difference were assessed.

Statistical analysis was done using the SAS ver. 9.3 (SAS Institute Inc., Cary, NC, USA) with statistical significance set at $\mathrm{p}<0.05$.

\section{Results}

When $2^{\circ}$ was taken as the acceptable margin of error with $96^{\circ}$ set as the standard $\alpha$ angle, there were 120 knees in group 1 and 34 knees in group 2. The femoral component was in neutral position $\left(96^{\circ}\right)$ in 7 knees, in varus position in 62 knees, and in valgus position in 85 knees.

Table 1. Preoperative and Postoperative Changes for Each Parameter

\begin{tabular}{clccc}
\hline Variable & Period & Number & Mean & p-value \\
\hline \multirow{2}{*}{ MAD } & Preoperative & 154 & 12.46 & $<0.001$ \\
& Postoperative & 154 & 1.66 & \\
PTA & Preoperative & 154 & 9.03 & 0.004 \\
& Postoperative & 154 & 10.47 & \\
\multirow{2}{*}{ KSKS } & Preoperative & 154 & 57.28 & $<0.001$ \\
& Postoperative & 154 & 96.10 & \\
\multirow{2}{*}{ KSFS } & Preoperative & 154 & 49.06 & $<0.001$ \\
& Postoperative & 154 & 84.68 & \\
\hline
\end{tabular}

Two sample t-test or Mann-Whitney test (Mann-Whitney test, if the p-value of the normality test was $<0.05$ in either group).

MAD: mechanical axis deviation, PTA: patellar tilt angle, KSKS: Knee Society Knee Score, KSFS: Knee Society Function Score.
The mean KSKS was improved from $57.3( \pm 15.9)$ preoperatively to $96.1( \pm 8.3)$ postoperatively and KSFS improved from $49.1( \pm 15.7)$ to $84.7( \pm 13.7)$ in all knees (Table 1$)$. The mean postoperative KSKS and KSFS were 96.0 and 84.5, respectively, in group 1 and 96.6 and 85.5, respectively, in group 2. There was no significant intergroup difference in the KSKS and KSFS ( $\mathrm{p}=0.67$ and $\mathrm{p}=0.56$, respectively) (Table 2 ).

The mean MAD was improved from $12.46^{\circ}( \pm 7.16)$ preoperatively to $1.66^{\circ}( \pm 3.36)$ postoperatively $(\mathrm{p}<0.001)$ (Table 1$)$ in all knees, specifically $1.59^{\circ}( \pm 3.28)$ in group 1 and $1.91^{\circ}( \pm 3.68)$ in group 2 ( $\mathrm{p}=0.63$ ) (Table 2). The number of outliers defined as $>2^{\circ}$ of MAD was not significantly different between the groups, 65 in group 1 and 24 in group $2(\mathrm{p}=0.12)$ (Table 3$)$. The mean PTA was $9.03^{\circ}( \pm 5.09)$ preoperatively and $10.47^{\circ}( \pm 5.69)$ at the last followup ( $\mathrm{p}=0.004)$ (Table 1). There was no significant difference in the PTA between the groups, $10.17^{\circ}( \pm 5.39)$ in group 1 and $11.58^{\circ}$ $( \pm 6.65)$ in group $2(\mathrm{p}=0.45)$ (Table 2$)$.

There was a negative correlation between the $\alpha$ angle and the $\operatorname{MAD}(\mathrm{p}=0.01$; correlation value, -0.20 ), whereas no correlation

Table 2. Postoperative Results for Each Parameter in Both Groups

\begin{tabular}{lccrcc}
\hline Variable & Group & Number & Mean & \multicolumn{1}{c}{ SD } & p-value \\
\hline MAD & 1 & 120 & 1.59 & 3.28 & 0.63 \\
& 2 & 34 & 1.91 & 3.68 & \\
PTA & 1 & 120 & 10.17 & 5.39 & 0.45 \\
& 2 & 34 & 11.58 & 6.65 & \\
KSKS & 1 & 120 & 95.96 & 9.09 & 0.67 \\
& 2 & 34 & 96.61 & 4.67 & \\
KSFS & 1 & 120 & 84.46 & 13.52 & 0.56 \\
& 2 & 34 & 85.45 & 14.60 & \\
\hline
\end{tabular}

Paired t-test or Wilcoxon signed rank test (Wilcoxon signed rank test, if the $\mathrm{p}$-value of the normality of difference was $<0.05$ ).

MAD: mechanical axis deviation, PTA: patellar tilt angle, KSKS: Knee Society Knee Score, KSFS: Knee Society Function Score.

Table 3. Comparisons of the Number of Outliers between Groups Divided According to Different a Angle Cut-off Values ${ }^{\text {a) }}$

\begin{tabular}{cccccc}
\hline \multirow{2}{*}{$\begin{array}{c}\text { Alpha angle } \\
\text { cut-off value }\end{array}$} & Group & \multicolumn{4}{c}{ Mechanical axis deviation outlier } \\
\cline { 3 - 6 } \pm 1 & $95 \leq \alpha<97$ & 44 & 1 & 31 & 1 \\
& $\alpha<95,97 \leq \alpha$ & 45 & & 31 & \\
\pm 2 & $94 \leq \alpha<98$ & 65 & 0.12 & 47 & 0.69 \\
& $\alpha<94,98 \leq \alpha$ & 24 & & 15 & \\
\pm 3 & $93 \leq \alpha<99$ & 84 & 1 & 58 & 1 \\
& $\alpha<93,99 \leq \alpha$ & 5 & & 5 & \\
\hline
\end{tabular}

${ }^{a)}$ Fishers' exact test. 
Table 4. Relationship between a Angle and Parameters

\begin{tabular}{llcc}
\hline \multicolumn{1}{c}{ Variable } & \multicolumn{1}{c}{ Method } & p-value & Correlation \\
\hline Mechanical axis deviation & Pearson's product-moment correlation & 0.01 & -0.20 \\
Patellar tilt angle & Spearman's rank correlation rho & 0.84 & -0.02 \\
Knee Society Knee Score & Spearman's rank correlation rho & 0.99 & -0.0003 \\
Knee Society Function Score & Spearman's rank correlation rho & 0.25 & 0.09 \\
\hline
\end{tabular}

Table 5. Comparisons of MAD between Groups Divided According to Different a Angle Cut-off Values ${ }^{\text {a }}$

\begin{tabular}{clc}
\hline Alpha angle cut-off value & Group & MAD (p-value) \\
\hline \pm 1 & $95 \leq \alpha<97$ & 0.28 \\
& $\alpha<95,97 \leq \alpha$ & \\
\pm 2 & $94 \leq \alpha<98$ & 0.85 \\
& $\alpha<94,98 \leq \alpha$ & \\
\pm 3 & $93 \leq \alpha<99$ & 0.12 \\
& $\alpha<93,99 \leq \alpha$ & \\
\pm 4 & $92 \leq \alpha<100$ & 0.47 \\
& $\alpha<92,100 \leq \alpha$ & \\
\end{tabular}

MAD: mechanical axis deviation.

a) -test.

was found with the other parameters (Table 4).

On the correlation between the MAD and the a angle, the cut-off value that yields the greatest difference in the MAD was $3^{\circ}$. Therefore, intergroup difference in the MAD was most distinctive when the patients were divided into a group with $93^{\circ}<\alpha$ angle $<99^{\circ}$ and the other group with an $\alpha$ angle of $<93^{\circ}$ or $>99^{\circ}$, although the difference was not at a statistically significant level $(\mathrm{p}=0.12)$ (Table 5).

\section{Discussion}

Proper component alignment is a crucial prognostic factor after TKA. This is because malalignment caused by improper bone resection results in component wear and loosening, which is the primary cause of TKA failure ${ }^{2,7-9)}$. Postoperative component alignment is essential for predicting the failure of TKA and the need for revision surgery.

Distal femoral resection in primary TKA is vital to achieving normal alignment in the coronal and sagittal planes. In particular, bone resection in the coronal plane has been considered as the most important procedure in $\mathrm{TKA}^{10)}$. Most of the contemporary TKA systems use intramedullary alignment guides along the anatomical axis of the femur. Femoral and tibial resections are performed separately in general and postoperative mechanical alignment depends on the accuracy of each bone resection. It has been thought that $2^{\circ}$ of deviation from the normal femorotibial angle on the coronal plane is acceptable $e^{8,9,11,12)}$. Studies have reported various important factors that are associated with femoral bone resection for accurate alignment on the coronal plane and the intramedullary alignment guide entry point is one of those. In this study, an intramedullary rod was inserted into the center of the femoral notch as opposed to medial insertion of a cutting guide. Medial insertion of the cutting guide has been associated with a varus alignment and lateral insertion with a valgus alignment. Reed and Gollish ${ }^{13)}$ suggested that the proper entry point of an intramedullary rod was $6.6 \mathrm{~mm}$ medial to the center of the femoral notch. Jiang and Insall ${ }^{14)}$ reported that rotation of the femur on the AP radiograph was associated with the knee alignment. However, such a relationship could be observable only when an excessive degree of femoral bowing was present and rotation tends to have no significant influence on the anatomical-mechanical axis. Accordingly, we believe that intramedullary guide insertion along the anatomical axis can be more effective in reducing malalignment error than the conventional insertion along the center of the femoral notch.

In this study, the intramedullary rod was inserted in $6^{\circ}$ valgus position to make the cutting guide be perpendicular to the mechanical axis of the femur, based on the assumption that the angular difference between the mechanical axis of the femur and the anatomical axis is $6^{\circ}$. However, the value can be different depending on the patient, in which a $6^{\circ}$ valgus insertion can result in malalignment. Accordingly, we measured the angle on the preoperative long leg AP radiograph in each patient and included the patients in whom the distal femoral cutting guide was perpendicular to the mechanical axis when inserted in $6^{\circ}$ valgus position in this study.

The length of an intramedullary rod which cannot be adjusted to the length of the femur results in errors due to the gap between the medullary canal and the rod. In our study, the number of knees with $\alpha>2^{\circ}$ of varus or valgus resection error in the coronal plane was 34 out of 154 (22\%), which was high compared to 24 out of 146 (16.4\%) in the study by Longstaff et al. ${ }^{6}$.

In particular, an intramedullary cutting guide needs to be 
inserted deeply for extensive femoral resection in knees with severe flexion contracture. During this procedure, the device we used in this study which using a hammer for cutting guide insertion instead of hands often results in a valgus knee because of applying valgus force on the femur even after the medial condyle of the distal femur in contact with the cutting guide. When the patients were divided with $96^{\circ}$ set as the standard $\alpha$ angle, more patients had valgus alignment (85 patients, $55.2 \%)$ than varus alignment. Thereafter, we have designed an intramedullary rod that is $2.5 \mathrm{~cm}$ longer than the traditional device with a length of $22 \mathrm{~cm}$ (Fig. 4).

In this study, we investigated the relationship between the $\alpha$ angle and the clinical and radiographic results and found a negative correlation between the $\alpha$ angle and the MAD. The greater the $\alpha$ angle was, the more valgus the femoral component position was, which eventually led to valgus alignment. Therefore, the cut off value of an acceptable $\alpha$ angle was determined based on the MAD.

Initially, the patients were divided into two groups for comparisons by taking $2^{\circ}$ as the acceptable margin of error of the $\alpha$ angle based on previous studies. However, there were no significant differences between the groups in the MAD, PTA, KSKS, and KSFS (MAD, $\mathrm{p}=0.63$; PTA, $\mathrm{p}=0.45$; KSKS, $\mathrm{p}=0.67$; KSFS, $\mathrm{p}=0.56$ ) (Table 2). Therefore, we investigated the cut-off value that would show intergroup differences in the MAD. When $3^{\circ}$ was taken as the cut-off value, or the patients were divided into those with $93^{\circ}>\alpha$ angle $<99^{\circ}$ and those with $\alpha$ angle $<93^{\circ}$ or $>99^{\circ}$, the difference in the $\mathrm{MAD}$ was most notable between the groups, which, however, did not show statistical significance $(\mathrm{p}=0.12)$ (Table 5).

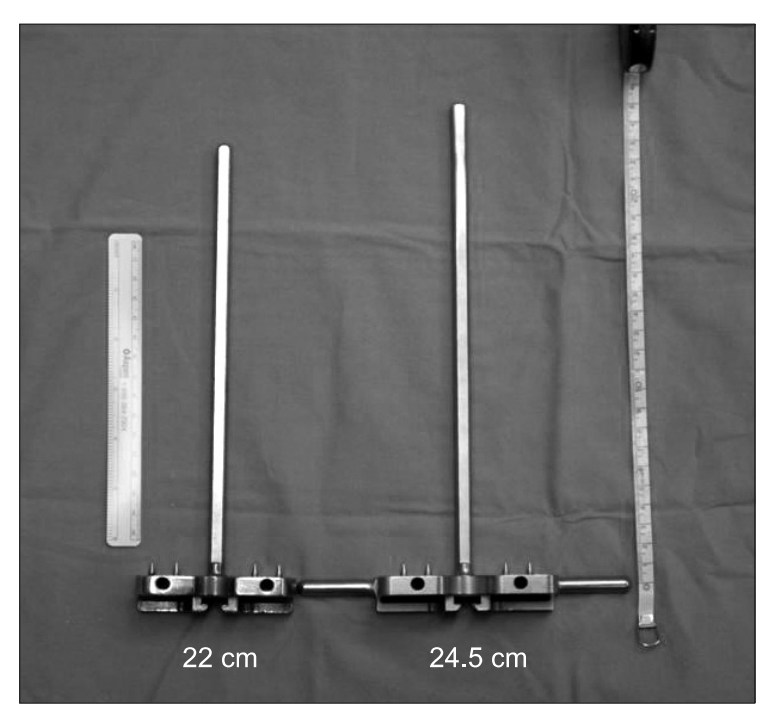

Fig. 4. A $22 \mathrm{~cm}$ intramedullary rod vs. a $2.5 \mathrm{~cm}$ extended rod.
Although the number of outliers with $>2^{\circ}$ MAD was higher in group 1 than group 2 (65 vs. 24) when grouping was done with $2^{\circ}$ chosen as the acceptable margin of error for $\alpha$ angle, there was no statistical significance in the difference $(\mathrm{p}=0.12)$ (Table 3). In addition, no significant intergroup difference was found in the number of outliers when other cut-off values were used for grouping (Table 3). We attributed this to the use of a device that enabled creation of a rectangular extension gap following soft tissue balancing, which allowed distal femoral cutting error to be partially compensated during proximal tibial cutting.

There was no statistically significant difference between the groups in the clinical outcome assessed by KSKS and KSFS. The PTA and MAD were not significantly different between the groups, which resulted in no remarkable difference in the coronal plane alignment. However, the greatest intergroup difference in the MAD could be observed when $3^{\circ}$ was taken as the cutoff value, which should be confirmed in a study with a longer follow-up period and a larger study population before clinical application.

We have made various attempts to improve the coronal plane alignment in IM-guided TKA. Currently, our method of choice is to use an intramedullary rod that is longer than the conventional rod in knees with the distance between the distal femur and the contact point between the femoral condyles is longer on the preoperative radiograph. In addition, we think that excessive varus or valgus resection can be prevented by predicting the proper thickness of the distal femoral resection based on the measurement of the length of a line that is tangent to the medial and lateral femoral condyles and perpendicular to the anatomical axis of the femur on the preoperative long leg plain AP radiograph.

There have been many studies on methods to reduce coronal plane alignment error and MAD. In particular, computer-aided navigation TKA has been reported effective in improving MAD in some studies ${ }^{15-17)}$.

The limitations of this study include that the study population was small $(n=154)$, radiographic assessment was done by one surgeon, and the follow-up period was short for establishing clinical end radiological outcome. Furthermore, we think that the influence of the relationship between the length of the femur and the intramedullary rod on the clinical and radiological outcome should be addressed in future studies.

\section{Conclusions}

In TKA using a fixed length intramedullary guide for femoral 
resection, the number of outliers with $>2^{\circ}$ of varus or valgus resection error in the coronal plane was relatively high (34 in 154 knees, 22\%). However, there was no statistically significant difference in clinical results between the knees with a resection error of $>2^{\circ}$ and those with $\leq 2^{\circ}$ (KSKS, $\mathrm{p}=0.67$; KSFS, $\mathrm{p}=0.56$ ).

\section{Conflict of Interest}

No potential conflict of interest relevant to this article was reported.

\section{References}

1. Tew M, Waugh W. Tibiofemoral alignment and the results of knee replacement. J Bone Joint Surg Br. 1985;67:551-6.

2. Ritter MA, Faris PM, Keating EM, Meding JB. Postoperative alignment of total knee replacement: its effect on survival. Clin Orthop Relat Res. 1994;(299):153-6.

3. Hungerford DS. Alignment in total knee replacement. Instr Course Lect. 1995;44:455-68.

4. Hungerford DS, Krackow KA. Total joint arthroplasty of the knee. Clin Orthop Relat Res. 1985;(192):23-33.

5. Sikorski JM. Computer-assisted revision total knee replacement. J Bone Joint Surg Br. 2004;86:510-4.

6. Longstaff LM, Sloan K, Stamp N, Scaddan M, Beaver R. Good alignment after total knee arthroplasty leads to faster rehabilitation and better function. J Arthroplasty. 2009; 24:570-8.

7. Moreland JR. Mechanisms of failure in total knee arthroplasty. Clin Orthop Relat Res. 1988;(226):49-64.

8. Townley CO. The anatomic total knee resurfacing arthro- plasty. Clin Orthop Relat Res. 1985;(192):82-96.

9. Cates HE, Ritter MA, Keating EM, Faris PM. Intramedullary versus extramedullary femoral alignment systems in total knee replacement. Clin Orthop Relat Res. 1993;(286):32-9.

10. Lotke PA, Ecker ML. Influence of positioning of prosthesis in total knee replacement. J Bone Joint Surg Am. 1977;59:779.

11. Insall JN, Binazzi R, Soudry M, Mestriner LA. Total knee arthroplasty. Clin Orthop Relat Res. 1985;(192):13-22.

12. Petersen TL, Engh GA. Radiographic assessment of knee alignment after total knee arthroplasty. J Arthroplasty. 1988; 3:67-72.

13. Reed SC, Gollish J. The accuracy of femoral intramedullary guides in total knee arthroplasty. J Arthroplasty. 1997;12:67782.

14. Jiang CC, Insall JN. Effect of rotation on the axial alignment of the femur: pitfalls in the use of femoral intramedullary guides in total knee arthroplasty. Clin Orthop Relat Res. 1989;(248):50-6.

15. Stulberg SD, Loan P, Sarin V. Computer-assisted navigation in total knee replacement: results of an initial experience in thirty-five patients. J Bone Joint Surg Am. 2002;84 Suppl 2:90-8.

16. Chauhan SK, Scott RG, Breidahl W, Beaver RJ. Computerassisted knee arthroplasty versus a conventional jig-based technique: a randomised, prospective trial. J Bone Joint Surg Br. 2004;86:372-7.

17. Sparmann M, Wolke B, Czupalla H, Banzer D, Zink A. Positioning of total knee arthroplasty with and without navigation support: a prospective, randomised study. J Bone Joint Surg Br. 2003;85:830-5. 